\title{
MODIFICACIÓN DE OLFATÓMETROS DE CUATRO BRAZOS PARA EXPERIMENTACIÓN EN BROCA DEL CAFÉ, Hypothenemus hampei FERRARI (COLEOPTERA: CURCULIONIDAE)
}

\author{
Arturo Pacheco ${ }^{1 / *}$,Helga Blanco-Metzler ${ }^{* *}$, Rebeca Mora* \\ Palabras clave: Broca del café, Hypothenemus hampei, olfatometría, olfatómetro de cuatro brazos, atrayente, \\ compuestos volátiles. \\ Keywords: Coffee berry borer, Hypothenemus hampei, olfactometry, four-arm olfactometer, attractant, volatile \\ compounds.
}

\section{RESUMEN}

La broca del café Hypothenemus hampei (Ferrari) es considerada la plaga de mayor importancia en el cultivo del café a nivel mundial. Al igual que muchos otros insectos, el comportamiento de la broca se ve modificado por distintas señales olfativas, lo cual ha impulsado la investigación en el campo de la olfatometría, por medio de una gran variedad de estrategias y alternativas. En este experimento se evaluaron 2 diseños innovadores de olfatómetros de flujo de aire de 4 vías para experimentación en $H$. hampei. Estos fueron fabricados totalmente en cristal y difieren entre ellos por la distribución angular de los brazos distribuidores de flujos de aire y la zona de ingreso y adaptación de los insectos al olfatómetro. Para evaluar la funcionalidad de estos diseños se utilizó, como atrayente de acción conocida, una mezcla de metanol-etanol 3:1 y se evaluó la cantidad de hembras adultas atraídas al tratamiento en cada olfatómetro. En el olfatómetro de $90^{\circ}$ los insectos se vieron claramente atraídos al brazo que contenía el atrayente, con un porcentaje del $45 \%$, mientras que el olfatómetro

\footnotetext{
1 Autor para correspondencia. Correo electrónico: arturo.pachecosolana@gmail.com

Centro de Investigación en Biología Celular y Molecular, CIBCM, Universidad de Costa Rica. San José, Costa Rica.
}

\begin{abstract}
Modification of four-arm olfactometers to be used with the coffee borer Hypothenemus hampei Ferrari (Coleoptera: Curculionidae). The coffee berry borer, Hypothenemus hampei is considered to be a major pest in coffee producing countries worldwide. As in many insects, the behavior of the coffee borer is modified through aromatic signals. This has triggered important research within the different approaches of olfactometry. Two new innovative designs of olfactometers were evaluated to be used on $H$. hampei for this research. The olfactometers were built of glass and differ in the angular distribution of their arms and in the insect's inlet and adaptation zone. A mixture of methanol-ethanol $3: 1$, with proven attraction properties, was used to evaluate the functionality of these olfactometers. The bioassays were performed using only adult females of $H$. hampei. On the $90^{\circ}$ olfactometer, $45 \%$ of the insects were strongly attracted to the arm comprised of the alcohol mixture. In the case of the $30^{\circ}$ olfactometer the positive behavior response was only of $1.5 \%$. During the
\end{abstract}

\footnotetext{
** Centro de Investigación en Protección de Cultivos, CIPROC, Universidad de Costa Rica. San José, Costa Rica.
} 
de $30^{\circ}$ tan solo alcanzó un 1,5\% de atracción. Las observaciones realizadas durante estas pruebas dejaron en evidencia que las estrategias de ingreso de los insectos a los olfatómetros y el área de ambientación influyeron de manera importante en estos resultados. En una segunda etapa se evaluó el olfatómetro de $90^{\circ}$, al comparar los promedios de atracción de cada uno de sus 4 brazos, y se verificó que las diferencias de atracción en cada brazo no fueron significativas $(\mathrm{p}<=0,05)$. Se concluye que el diseño del olfatómetro de $90^{\circ}$ es apropiado para realizar pruebas de atracción a compuestos volátiles en hembras adultas de H. hampei.

\section{INTRODUCCIÓN}

El café es uno de los cultivos de mayor importancia económica en una gran cantidad de países tropicales. Hoy en día es cultivado en más de 60 países (Waller et al. 2007) con un estimado anual de ventas al detalle de más de $\$ 70$ mil millones (Vega et al. 2006). Alrededor de 25 millones de productores dependen del cultivo de café como medio de sustento (Waller et al. 2007).

En muchos países la producción de café está seriamente amenazada por diferentes plagas y enfermedades; entre estas se encuentra la broca del café, Hypothenemus hampei (Ferrari) (Coleoptera: Curculionidae) la cual es una seria plaga en prácticamente todas las regiones productoras de café con la excepción del archipiélago Hawaiano (Vega et al. 2006).

La broca del café al igual que la gran mayoría de insectos se ve atraída por diversas señales olfativas. Las investigaciones sobre atracción olfativa de los insectos plaga, por compuestos volátiles liberados desde sus plantas hospederas, son cada vez mas frecuentes (Dickens 1999). Todos estos estímulos olfativos tienen un potencial importante en el desarrollo de técnicas biológicas para el control de plagas (Sengonca y Kranz 2001 ). assays it was observed that the different insect insertion strategies into the olfactometers and the adaptation area had a major influence on results. During the second stage of this research, the $90^{\circ}$ olfactometer was evaluated through a comparison of the average attraction of each of its 4 arms; there were no significant differences $(\mathrm{p}<=0.05)$ in attraction response of $H$. hampei between arms of the device. It is concluded that the $90^{\circ}$ olfactometer design is suitable to develop volatile-compound attraction assays on adult females of $H$. hampei.
La investigación en olfatometría para la broca del café ha sido poca y enfocada hacia trampeo en campo (Borbón y Mora 2000). Los estudios de laboratorio se han realizado con varios tipos de olfatómetros, adaptándolos a las necesidades de cada investigación pero sin comprobar su eficiencia con respecto a las características y comportamiento del insecto (Mendesil et al. 2009).

Existen 2 tipos generales de diseño para los olfatómetros: los de aire estático y los de flujo de aire. Los olfatómetros de aire estático se usan para medir respuestas quimiotáctiles u olfativas a corta distancia o en situaciones en donde las señales químicas se difunden en el espacio sin ser transportadas por una corriente de aire. Los de flujo de aire se usan en estudios encaminados a la detección y medición de respuestas de atracción de insectos a señales olfativas emitidas en distancias largas y medianas, ya que permite evaluar conductas de anemotaxis desencadenadas por aromas contenidos en una corriente de aire (López-Ávila y Rincón 2006).

Los olfatómetros de flujo de aire deben utilizar un sistema que permita mantener un flujo continuo del aire dentro del dispositivo. Existen 3 modelos básicos en este tipo de olfatómetro: túnel de viento, tubo en ' $\mathrm{Y}$ ' y de 4 vías. El olfatómetro 
de túnel de viento tiene la virtud de contar con una cámara de decisión amplia especialmente útil para insectos con capacidad de vuelo. $\mathrm{Su}$ deficiencia se encuentra en que sólo permite evaluar un compuesto a la vez. Los olfatómetros en $\mathrm{Y}$ permiten empezar a discernir elecciones por parte del insecto al existir 2 opciones de ruta, pero han presentado algunos problemas en el manejo de los flujos de aire. El primer diseño de un olfatómetro de flujo de aire de 4 vías fue desarrollado por Pettersson (1970) para estudiar respuestas de áfidos a feromonas sexuales; estos permiten observar relaciones más complejas de atracción y repelencia a varios compuestos en un solo experimento. Otra ventaja es la posición en que puede ser ingresado el insecto justo en el centro de los flujos de aire, pero en general tienen la deficiencia de tener campos de decisión muy pequeños y normalmente son más utilizados para pruebas en las que sólo se usa un insecto y se registra el tiempo en que el insecto se mueve sobre cada zona del olfatómetro.
En este trabajo se presentan 2 diseños innovadores de olfatómetros de flujo de aire de 4 vías, fabricados totalmente en cristal para evitar cualquier persistencia de compuestos aromáticos en sus paredes. Se incluye además, 2 distribuciones diferentes de los brazos por donde ingresan los aromas, al igual que 2 estrategias diferentes de ingreso de los insectos al olfatómetro.

La combinación de estas diferentes características en ambos diseños, son las que se evaluaron mediante el uso de un compuesto de atracción comprobada sobre la broca, para así poder determinar cual olfatómetro tiene el mejor desempeño y utilizarlo posteriormente en nuevas pruebas olfatométricas en $H$. hampei.

\section{MATERIALES Y MÉTODOS}

Con el fin de validar el desempeño de 2 dispositivos olfatométricos para broca del café se evaluaron modificaciones de 2 olfatómetros de 4 vías (Figura 1) descritos por Morales (1989)

\section{Olfatómetro de $30^{\circ}$}

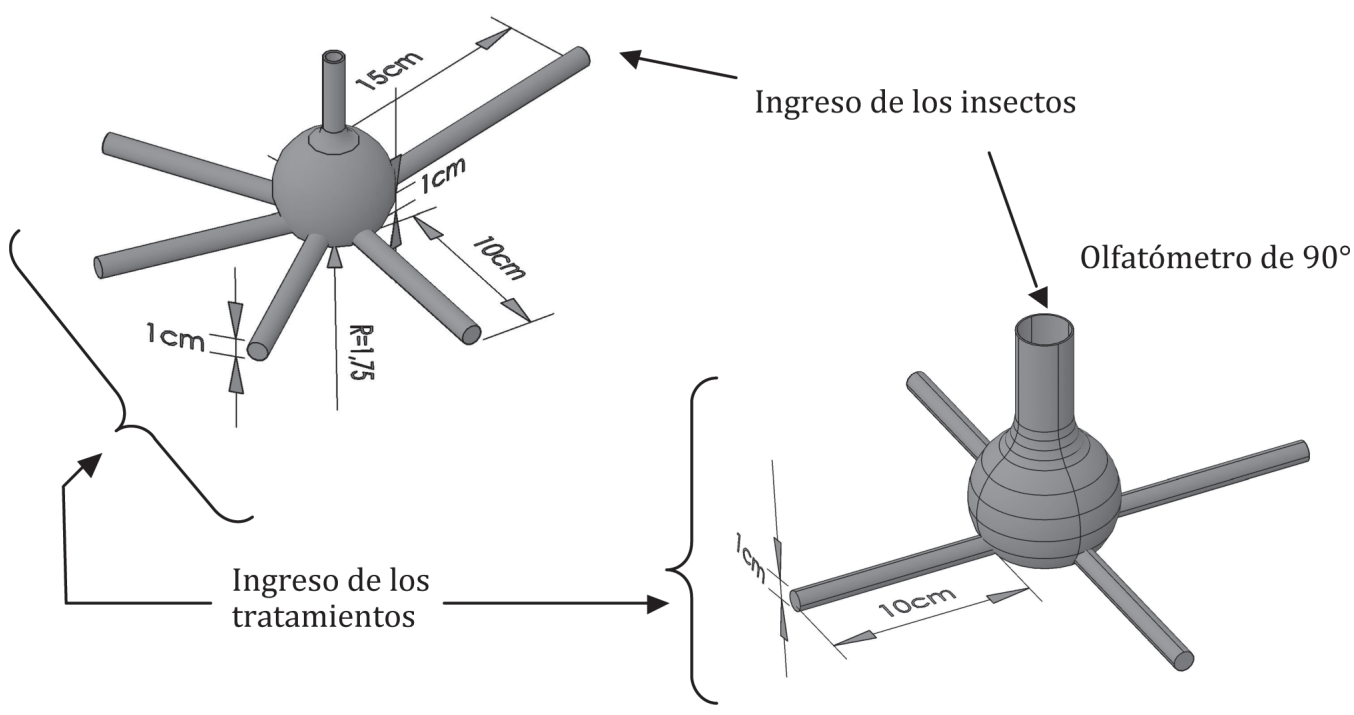

Fig. 1. Dos diseños innovadores de olfatómetros puestos a prueba con un atrayente de acción comprobada sobre H. hampei. 
y López-Ávila y Rincón (2006). Se realizaron pruebas olfatométricas con compuestos volátiles de acción conocida y comprobada, en este caso se utilizó la mezcla de metanol y etanol en proporción 3:1 utilizada en investigaciones sobre trampeo (Borbón y Mora 2000) y específicamente se usó la mezcla comercial que actualmente distribuye el CICAFE (Centro de Investigaciones en Café) entre los productores de café en Costa Rica, para usar en trampas de campo. Se evaluó la cantidad de insectos atraídos al tratamiento y se determinó cual olfatómetro presentaba un mejor desempeño.

Los insectos utilizados en los bioensayos se obtuvieron de la colonia que actualmente se mantiene en dieta artificial en el insectario del Centro de Investigaciones en Biología Celular y Molecular (CIBCM) de la Universidad de Costa Rica. Esta colonia de broca de café se desarrolló con los parámetros de cría descritos por Obando (2002) al igual que las modificaciones de la dieta artificial tipo Cenibroca II recomendados por Portilla y Streett (2008). Para la reproducción de los insectos se utilizan placas multipozo de 24 pozos marca Evergreen, donde se dispensa la dieta artificial y se colocan 4 hembras de $H$. hampei por pozo. Las placas se colocan en cajas plásticas que representan un lote y una generación. Todos los lotes se mantienen en una incubadora a $80 \%$ de humedad relativa y $27^{\circ} \mathrm{C}$. La población de insectos se renueva y mantiene la suficiente variabilidad genética con ingresos de nuevos individuos cada 3 meses. Estos provienen de la colonia en grano de campo del CICAFE en Barva de Heredia, la cual se mantiene en constante regeneración con material infestado de diversas fincas del país.

Para realizar los bioensayos se utilizaron exclusivamente hembras adultas colonizadoras. Estas hembras son aquellas que salen por si mismas del pozo con dieta en busca de un nuevo sitio apto para ovopositar. Estas hembras se recolectan fácilmente pues en cada lote se coloca un pliego de papel toalla en el fondo, al cual estas hembras se adhieren.

El área mecánica y el sistema de distribución de aromas de los olfatómetros se basó en la utilizada por Sengonca y Kranz (2001) y Mendesil et al. (2009) y se presenta de forma esquematizada en la Figura 2. El atrayente se hizo

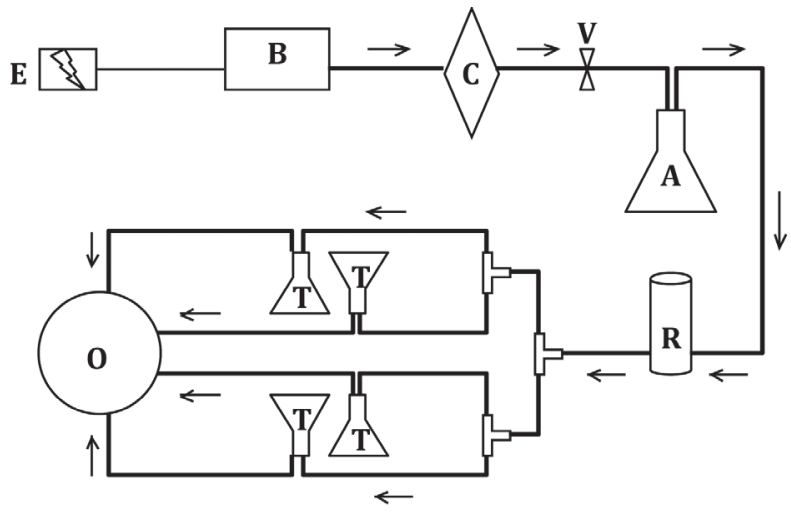

$\rightarrow$ Dirección del flujo de Aire
E Fuente de poder
B Bomba de aire
C Filtro de carbón activado

V Válvula de paso

A Trampa de agua destilada

R Rotámetro

T Fuente aromática

0 Olfatómetro (ambos)

Fig. 2. Representación esquematizada de todos los componentes de ambos olfatómetros de 4 brazos modificados. 
llegar al olfatómetro con ayuda de una bomba de aire marca Barnant Co. Modelo 400-3901 (115 vac, $60 \mathrm{~Hz}$ ) que impulsó una corriente de aire hasta ser forzada a pasar a través de un filtro de carbón activado, para posteriormente pasar a través de una trampa de agua destilada para filtrar cualquier impureza restante y agregar humedad a la corriente de aire. Por último, se colocó un rotámetro para medir el flujo de aire y 3 piezas $T$ para separar en 4 flujos iguales la corriente de aire, hacia cada uno de los tratamientos en erlenmeyers de $125 \mathrm{ml}$ y posteriormente al olfatómetro.

Se modificaron los diseños originales de la zona central del olfatómetro, donde se colocaron a los insectos para adecuarse al tamaño de $H$. hampei y facilitar la observación de los individuos durante los bioensayos. La cámara central se amplió principalmente en altura $(5 \mathrm{~cm}$.) para permitir que el insecto vuele con mayor libertad en busca de la preferencia aromática. También los brazos fueron diseñados con $10 \mathrm{~cm}$. de longitud para asegurar la decisión del insecto una vez dentro del brazo. La pieza central de los olfatómetros se fabricó como una sola pieza de vidrio para evitar la adherencia de compuestos aromáticos en su estructura. Ambos dispositivos fueron diferenciados en su nombre por el ángulo de inserción de sus brazos.

\section{Prueba olfatométrica}

Con base en un sistema similar al utilizado por Mendesil et al. (2009), cada experimento tuvo una duración de $16 \mathrm{~min}$, con alternancia en la posición del atrayente cada vez que se iniciaba una nueva sesión experimental. El flujo de aire se calibró en $200 \mathrm{ml} . \mathrm{s}^{-1}$ según las recomendaciones de Sengonca y Kranz (2001) sobre flujos de aire máximos, mínimos e ideales para insectos de tamaño pequeño.

El área de trabajo se cubrió por todos sus costados con malla aluminizada de tipo Aluminet ${ }^{\circledR} 50 \%$, para evitar estímulos visuales no deseados, gracias al efecto de difusión y homogenización de la luz que produce el tejido torneado de este material. La iluminación se realizó de abajo hacia arriba con 6 lámparas fluorescentes de luz blanca cubiertas por una lámina de papel pergamino blanco translúcido para conseguir un efecto de luz difusa.

Los insectos se mantuvieron primero por 5 min en el área de ambientación para luego dejarlos libres en el dispositivo. En ese momento se abrían los flujos de aire con el respectivo atrayente. En el olfatómetro de $90^{\circ}$ el área de ambientación consistía en un tubo de cristal, de diámetro apenas menor que la columna central del olfatómetro, el cual se introducía por esta última para así colocar a los insectos directamente en el fondo de la cámara central del olfatómetro. Al pasar el tiempo de ambientación simplemente se retiraba el tubo y las brocas quedaban libres dentro del dispositivo. En el caso del olfatómetro de $30^{\circ}$ el área de ambientación consistía en una pieza plástica con forma de codo cuyo extremo inferior se acoplaba al brazo de ingreso de los insectos. En la unión de ambas piezas se colocó una malla de tergal que era retirada fácilmente pasados los 5 min de ambientación para que los insectos quedaran libres frente a la corriente de aire del olfatómetro.

Debido al alto costo de la pieza central de cristal del olfatómetro sólo se contó con una y por lo tanto se procedió a aleatorizar las secuencias de tratamientos y repeticiones. De igual manera esta pieza de cristal se lavó con alcohol de $95 \%$ y acetona (grado técnico para HPLC) entre repeticiones para eliminar cualquier rastro aromático que pudiera quedar entre experimentos. Adicionalmente dicha pieza se autoclavó durante 25 min, entre las distintas pruebas, junto con el resto de piezas de cristal y manguerillas que conformaron todo el sistema del olfatómetro.

\section{Diseño experimental y análisis estadístico}

Para los experimentos en cada uno de los olfatómetros se usó un diseño irrestricto al azar. Se realizaron 3 repeticiones en cada uno de los brazos de ambos olfatómetros, considerándolos como 2 tratamientos diferentes a causa de los ángulos de inserción de sus brazos y se utilizaron 50 individuos por repetición. La variable de respuesta fue el ingreso permanente del insecto a la 
zona exclusiva del tratamiento, los insectos que transcurridos los 16 min del tiempo estipulado se mantenían en la cámara central se les asignaba el estado de SR (sin respuesta).

Para el análisis estadístico se utilizaron los conteos de brocas observadas en los brazos de la cámara principal del olfatómetro. Se obtuvo el total de brocas en cada uno de los brazos, se promediaron y se efectuó un ANDEVA (análisis de variancia) para cada experimento. También se realizó una prueba LSD Fisher para encontrar diferencias significativas entre los promedios de los tratamientos. El análisis se realizó con el software Infostat ${ }^{\circledR}$.

\section{RESULTADOS}

Las hembras adultas de la broca del fruto del café presentaron comportamientos claramente contrastantes entre ambos diseños de olfatómetros. La Figura 3 muestra como en el olfatómetro de $90^{\circ}$ los insectos se vieron fuertemente atraídos al brazo que contenía el atrayente de etanol-metanol, con un promedio cercano a los 24 individuos, mientras que en el olfatómetro de $30^{\circ}$ este promedio apenas alcanzó los 3 individuos. También se pudo observar que en ambos dispositivos hubo un promedio alto de insectos que no presentaron respuesta al tratamiento, esto quiere decir que al terminar el tiempo de tratamiento los insectos se encontraban dentro de la cámara central de los olfatómetros o en el área de ingreso. La mayoría de los individuos "sin respuesta" no presentaron comportamiento de vuelo y en general caminaban hacia arriba por la columna superior que permite la salida de las corrientes de aire en ambos dispositivos. En el caso particular del olfatómetro de $30^{\circ}$, muchos insectos se quedaron inmóviles en la unión del dispositivo de ambientación y el brazo de ingreso del olfatómetro. Por su parte en la Figura 3 también se puede observar que los resultados obtenidos por el olfatómetro de $90^{\circ}$ indican que el número promedio de individuos atraídos por el tratamiento (una media de 24) fue mayor que aquellos que no presentaron ninguna respuesta (alrededor de 17 individuos).

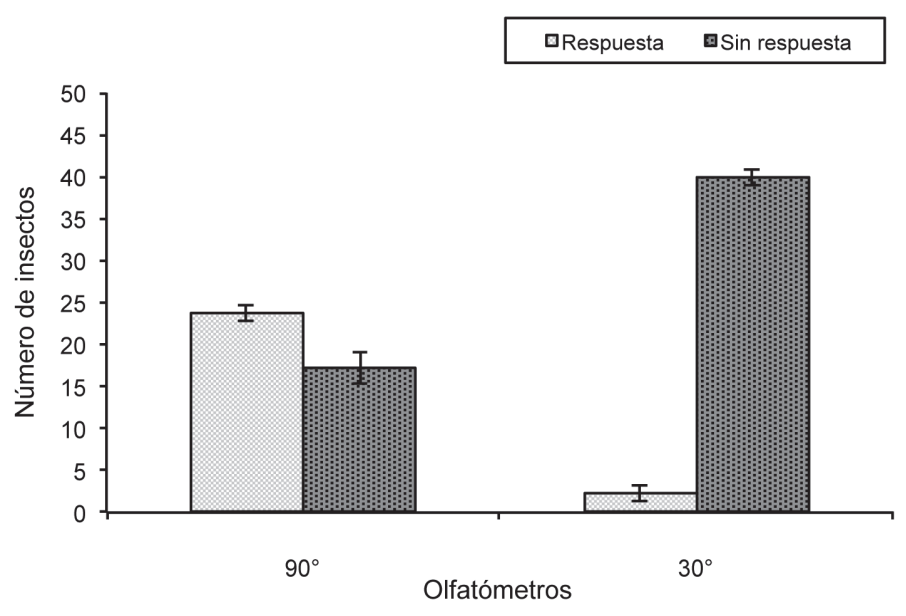

Fig. 3. Comparación de la respuesta olfativa de hembras adultas de Hypothenemus hampei en 2 diseños de olfatómetros con una mezcla de etanol-metanol como atrayente. 
En el Cuadro 1 se muestra en detalle el comportamiento de las hembras adultas de $H$. hampei en cada uno de los brazos de ambos dispositivos olfatométricos. En el olfatómetro de $90^{\circ}$ la respuesta de la broca en los brazos a los que sólo se aplicó una corriente de aire sin atrayente fue mínima $(\approx 3)$ y sin diferencias significativas entre estos $(\mathrm{F}=2,57 ; \mathrm{p}=0,184)$. Por su parte el brazo de este olfatómetro en que fue aplicado el atrayente sí mostró un promedio alto de atracción del insecto $(\approx 24)$ y significativamente diferente al promedio de brocas que no presentaron respuesta, tal como lo muestra el análisis de varianza con valores de 27,68 y 0,0063 para $\mathrm{F}$ y p respectivamente. En el caso del olfatómetro de $30^{\circ}$ ninguno de los 4 brazos presentaron una respuesta considerable por parte de la broca $(<3)$ y ninguno fue significativamente diferente entre sí $(\mathrm{F}=1,88 ; \mathrm{p}=0,241)$.

La Figura 4 toma en cuenta nada más los 4 brazos del olfatómetro de $90^{\circ}$ tratados individualmente con la mezcla atrayente de etanol-metanol. Los resultados mostraron que cada brazo logró atraer entre 20 y 25 individuos en promedio cuando se hizo pasar el atrayente por cada uno de ellos y no se detectaron diferencias significativas entre ninguno de los brazos $(\mathrm{F}=0,35 ; \mathrm{p}=0,788)$.

Cuadro 1. Promedio de hembras adultas de Hypothenemus hampei atraídas por la mezcla de etanol-metanol en 2 olfatómetros de 4 brazos.

\begin{tabular}{cccccc}
\hline & & \multicolumn{2}{c}{ Tratamientos } & & \\
Olfatómetro & Etanol-metanol & Aire & Aire & Sin respuesta \\
\hline $90^{\circ}$ & $23,6 \mathrm{a}$ & $3,5 \mathrm{~b}$ & $2,6 \mathrm{~b}$ & $3 \mathrm{~b}$ & $17,2 \mathrm{c}$ \\
$30^{\circ}$ & $2,3 \mathrm{~b}$ & $2,4 \mathrm{~b}$ & $2,5 \mathrm{~b}$ & $2,9 \mathrm{~b}$ & $39,8 \mathrm{a}$ \\
\hline
\end{tabular}

Letras distintas indican diferencias significativas $(\mathrm{p}<=0,05)$.

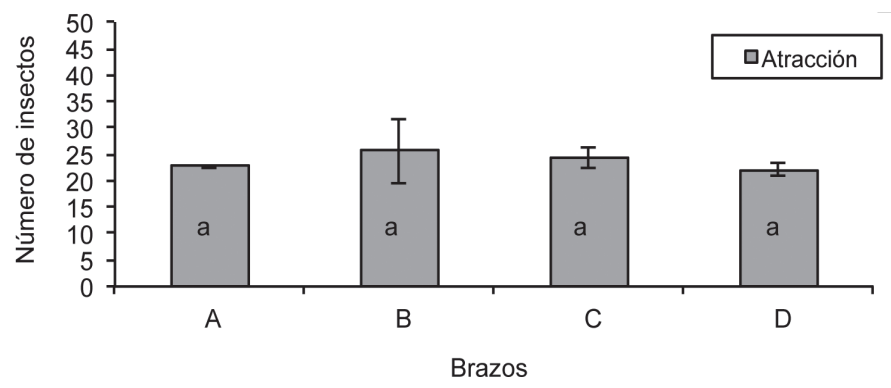

Columnas con la misma letra no presentan diferencias significativas $(\mathrm{p}<=0,05)$

Fig. 4. Comparación de la capacidad de atracción olfativa de cada brazo del olfatómetro de $90^{\circ}$ en hembras adultas de Hypothenemus hampei expuestas a una mezcla de etanol-metanol.

\section{DISCUSIÓN}

Los 2 diseños de olfatómetro desarrollados en el presente estudio son variantes modificadas de los diseños descritos por Morales (1989) y López-Ávila y Rincón (2006). Estas modificaciones, basadas en las características físicas y biológicas del insecto a estudiar (H. hampei) y observaciones en experimentos preliminares, son importantes para optimizar futuros experimentos en el campo de la olfatometría en broca del café.

Para diseñar estos modelos de olfatómetro se tomaron en cuenta las fortalezas y debilidades de los 3 diferentes tipos de olfatómetro.

Para los modelos de olfatómetro presentados en este estudio, se amplió la cámara central 
a fin de permitir suficiente movimiento de exploración y decisión a los 50 individuos que se usaron en cada repetición. Ese espacio ampliado además evitó la formación de turbulencia en el área de decisión y además la longitud de los brazos permitió evaluar con mayor claridad la respuesta olfativa de los insectos. El criterio más utilizado en la determinación de un compuesto volátil en olfatómetros de tipo $\mathrm{Y}$, es la distancia que el insecto camina dentro del brazo tratado (Dean y Satasook 1983). No obstante, en esta investigación la reacción ante un olor particular fue medida por la cantidad de individuos atraídos al brazo del olfatómetro bajo tratamiento y que se mantuvieron dentro de ese sector.

Durante la fase preliminar de esta investigación, se observó que la luz y otras influencias exteriores, como lo son distintos colores, resplandores o sombras, son capaces de afectar el comportamiento de los individuos bajo experimentación. Para evitar estas fuentes de error, el espacio donde se realizaron los experimentos se cubrió en todos sus lados además de la parte superior también la inferior con malla aluminizada y la fuente de luz se colocó en el plano inferior cubierta por un pliego de papel pergamino para hacerla difusa. Otros investigadores han utilizado técnicas similares; Venzon et al. (1999) utilizó una cubierta de forma triangular sobre un olfatómetro en Y. Por su parte Geier y Boeckh (1999) utilizaron láminas de cartón blanco a cada costado de un olfatómetro en Y para evitar los estímulos visuales no deseados, pero este método puede traer inconvenientes como la formación de sombras o gradientes de luz que pueden afectar el comportamiento de los insectos. La iluminación constante y difusa utilizada en este estudio también es recomendada por otros autores (Colazza et al. 1999).

Es importante destacar que todos los experimentos fueron realizados con hembras adultas colonizadoras. Esto quiere decir que son individuos recolectados fuera de la dieta artificial, las cuales según Mathieu et al. (2001) presentan comportamiento similar a las hembras colonizadoras en campo y difieren del comportamiento de aquellas hembras que se mantiene dentro del fruto en campo o dentro de la dieta artificial en laboratorio, las cuales muestran poca respuesta a estímulos olfativos. Estos individuos que se adhieren al papel toalla que cubre el fondo de las cajas con cada lote de la colonia de broca, son ideales para experimentación pues son hembras cargadas de huevos cuyos instintos de búsqueda de frutos de café ideales para la ovoposición se encuentran muy activos.

Para ambos diseños de olfatómetros descritos en este estudio se obtuvieron resultados muy contrastantes. El olfatómetro de $90^{\circ}$ produjo resultados satisfactorios al atraer cantidades considerables (45\%) de insectos al brazo en el cual se colocaba el atrayente, mientras que el olfatómetro de $30^{\circ}$ tuvo un desempeño insuficiente al quedar la mayoría de los individuos prueba $(80 \%)$ en el brazo de ingreso o la cámara central. Estos resultados pueden deberse a una de las principales diferencias en estudio entre ambos diseños: el método de ingreso de los individuos, el cual se realizaba por un quinto brazo horizontal en el olfatómetro de $30^{\circ}$ al colocar a los individuos directamente en el centro de la cámara de vidrio en el diseño de $90^{\circ}$. En este último, el mecanismo de ingreso de los insectos permite que estos sean simultáneamente expuestos a los 4 flujos de aire de cada brazo, lo cual, algunos autores indican que es fundamental en experimentos de olfatometría (Rapusas et al. 1996). Gonzales et al. (1999) describe un área central en un olfatómetro de 4 brazos para parasitoides pero no explica cómo se realiza el ingreso de los individuos prueba. Por su parte Ponsonby y Copland (1995) usaron una pequeña cámara la cual permitió un "ingreso sin estrés para los insectos". En este estudio, el diámetro del conducto superior de salida de los flujos de aire del olfatómetro de $90^{\circ}$, permitía introducir un tubo de vidrio directamente en el centro de la cámara central del olfatómetro donde los insectos se alojaban en un área de ambientación durante 5 min, luego sencillamente se retiraba el tubo y los individuos prueba quedaban libres y expuestos a los 4 flujos de aire. Por su parte el olfatómetro de $30^{\circ}$ contaba con un quinto brazo, opuesto a 
los que conducen los tratamientos, por donde se realizaba el ingreso de los insectos. A este brazo se le tuvo que diseñar una pieza adicional, similar a un codo de $90^{\circ}$ para facilitar el ingreso de los insectos y a su vez desempeñar la función de área de ambientación durante los 5 min previos a la exposición al atrayente. Se observó que en el momento de liberar los insectos en el flujo de aire muchos se alojaban en la unión entre el brazo y el dispositivo de ingreso. Esto responde a la naturaleza innata de $H$. hampei que busca la rugosidad de cualquier superficie ya que en el fruto de café sus sitios de penetración son principalmente la corona o en menor medida el pedúnculo. Aún así, los insectos que alcanzaban la cámara central del olfatómetro y sus brazos no mostraron atracción al tratamiento de etanol-metanol.

Una vez determinado cual modelo de olfatómetro presentaba resultados prometedores se debió verificar que no hubiera diferencias significativas en la capacidad de atracción de cada brazo cuando se le colocaba el atrayente. En el caso de la broca del café, como ya se mencionó, era indispensable que la superficie donde se moviera el insecto fuere lo más uniforme posible y por eso resulta una gran ventaja que el olfatómetro consista de una sola pieza de vidrio. Al conducir el flujo de aire con el atrayente, se compararon los promedios de atracción de cada brazo del olfatómetro de $90^{\circ}$ y se pudo verificar que las diferencias no son significativas y por lo tanto una simple aleatorización de los tratamientos en futuras investigaciones permitirá obtener datos fiables. Es importante recalcar que la adaptación de todo el espacio donde se realicen las pruebas es necesaria para evitar las distracciones lumínicas y visuales que ya se comentaron. Algunos autores para tratar este problema recomiendan que durante la prueba olfatométrica el olfatómetro de 4 brazos sea girado un cuarto de vuelta cada determinado tiempo (Mendesil et al. 2009, Vet et al. 1983).

Esta investigación demostró que el olfatómetro modificado de 4 brazos a $90^{\circ}$ descrito en el presente estudio, es apropiado para realizar pruebas de atracción a compuestos volátiles en hembras adultas de $H$. hampei.

\section{LITERATURA CITADA}

BORBÓN O., MORA O. 2000. Proyecto de trampas, atrayentes y repelentes para el control de la broca del fruto del cafeto. In: XIX Simposio Latinoamericano de Caficultura, Costa Rica, 2000, p. 331-348

COLAZZA S., SALERNO D., WAJNBERG E. 1999. Volatile and contact chemicals released by Nezara viridula (Heteroptera: Pentatomidae) have a kairomonal effect on the egg parasitoid Trissolcus basalis (Hymenoptera: Scelionidae). Biological Control 16:310-317.

DEAN G.J., SATASOOK C. 1983. Response of Chrysoperla carnea (Stephens) (Neuroptera: Chrysopidae) to some potential attractants. Bull. Ent. Res. 73:619624.

DICKENS J.C. 1999. Predatory-prey interactions: olfactory adaptions of generalist and specialists predators. Agric. Forest Entom. 1:47-54.

GEIER M., BOECKH J. 1999. A new Y-tube olfactometer for mosquitoes to measure the attractiveness of host odours. Entomol. Exp. Appl. 92:9-19.

GONZALES W.L., FUENTES-CONTRERAS E., NIEMEYER H.M. 1999. Semiochemicals associated to spacing behaviour of the bird cherry-oat aphid Rhopalosiphum padi L. (Hem. Aphididae) do not affect the olfactometric behaviour of the cereal aphid parasitoid Aphidius rhopalosiphi De Stephani-Perez (Hym. Braconidae). J. Appl. Ent. 123:413-415.

LÓPEZ-ÁVILA A., RINCÓN D.F. 2006. Diseño de un olfatómetro de flujo de aire para medir respuestas olfativas de insectos de tamaño mediano y pequeño. Revista Corpoica-Ciencia y Tecnología Agropecuaria 7(1):61-65.

MATHIEU F., GAUDICHON V., BRUN L.O., FREROT B. 2001. Effect of physiological status on olfactory and visual responses of female Hypothenemus hampei during host plant colonization. Physiological Entomology 26:189-193.

MENDESIL E., BRUCE T.J.A., WOODCOCK C.M. 2009 Semiochemicals used in host location by the coffee berry borer, Hypothenemus hampei. Journal of Chemical Ecology 35:944-950.

MORALES H.E. 1989. Atracción y colonización de Anthonomus eugenii Cano a diferentes solanáceas hospederas: posibilidad de control cultural en chile dulce. CATIE. Turrialba, Costa Rica. 95 p.

OBANDO A. 2002. Levantamiento de un pie de cría de broca del café, Hypothenemus hampei (Coleóptero, Scolytidae) en granos de café y en dieta artificial. 
Tesis: Ingeniero Agrónomo. Instituto Tecnológico de Costa Rica, San Carlos, Costa Rica. 76 p

PETTERSON J. 1970. An aphid sex attractant. I. Biological studies. Entomol. Scandinavica 1:63-73.

PONSONBY D.J., COPLAND J.W. 1995. Olfactory responses by the scale insect predator Chilocorus nigritus (F.) (Coleoptera: Coccinellidae). Biocontrol Sci. Techn. 5:83-93.

PORTILLA M., STREETT D. 2008. Avances investigativos en la producción masiva automatizada de Broca del Café Hypothenemus hampei Ferrari (Coleoptera: Scolytidae) y de sus parasitoides sobre dietas artificiales. Sistemas Agroecológicos y Modelos Biomatemáticos 1 (1). Julio 2008.

RAPUSAS H.R., BOTTRELL D.G., COLI M. 1996. Intraespecific variation in chemical attraction of rice to insect predators. Biological Control 6, 394-400.
SENGONCA C., KRANZ A. 2001. A modified, four-armed olfactometer for determining olfactory reactions of beneficial arthropods. Journal of Pest Science 74:127-132.

VEGA E., POSADA F., INFANTE F. 2006. Coffee Insects: Ecology and Control. Encyclopedia of Pest Management. pp. 1-4.

VENZON M., JANSSENS A., SABELIS M.S. 1999. Attraction of a generalist predator towards herbivoreinfested plants. Entomol. Exp. Appl. 93:305-314.

VET L.M., LENTEREN J.C., HEYMANS M., MEELIS E. 1983. An airflow olfactometer for measuring olfactory responses of hymenopterous parasitoids and other small insects. Physiol. Entomol. 8:97-106.

WALLER J.M., BIGGER M., HILLOCK R.A. 2007. Coffee Pests Disease and their Management. CAB International, Egham, Surrey, UK. 434 p. 